\title{
SOFT X-RAY EMISSION OF QUASARS
}

\author{
N. SCHARTEL, R. WALTER, H.H. FINK \\ Max Planck Inst. für extraterrestr. Physik, Giessenbachstraße, D-85748 Garching b. München
}

\begin{abstract}
From a list of known quasars compiled from various catalogues we selected all sources detected by the PSPC $(0.1-2.4 \mathrm{keV})$ aboard ROSAT with more than 80 counts during the all sky survey. A sample of 102 sources resulted. At higher redshifts most of the selected sources are radio-loud. At a redshift smaller than 0.50 we found 54 radio-quiet quasars and 30 radio-loud sources. For this reduced sample the mean spectral index of the radio-quiet sources $(\langle\Gamma\rangle=2.53)$ and that of the radio-loud ones $(\langle\Gamma\rangle=2.26)$ are clearly different with a significance of $3.3 \sigma$.

About 2/3 of the bright quasars observed with Einstein also belong to our sample. The spectra observed with ROSAT are sytematically steeper than the ones observed with Einstein yielding a $<\Gamma_{R O S A T}-\Gamma_{\text {Einstein }}>$ of $0.66 \pm 0.18$ for radio quiet and of $0.68 \pm 0.19$ for radio-loud sources, respectively.

For radio loud quasars, the mean spectral slope decreases from 2.3 to 1.5 when the redshift increases beyond 0.5 (figure 1). The fact that high redshift sources show a photon index of about 1.5, which is similar to the mean index observed with Einstein for radio-loud sources, suggests that this decrease towards higher redshifts can be interpreted by the shift of the soft $\mathrm{X}$-ray excess outside of the ROSAT spectral band when the redshift increases. The solid lines in figure 1 represent theoretical pathes of the photon index as a function of the redshift as derived from simulations assuming a power law plus black body model spectrum for the quasars X-ray emission. In curve No 1 the powerlaw index is fixed to 1.4. To be compatible with the observation the temperature of the blackbody component must range between 50 and $70 \mathrm{eV}$. Curve No 2 asssumes the same model with a powerlaw index fixed to 1.8 to account for radio quiet sources.
\end{abstract}

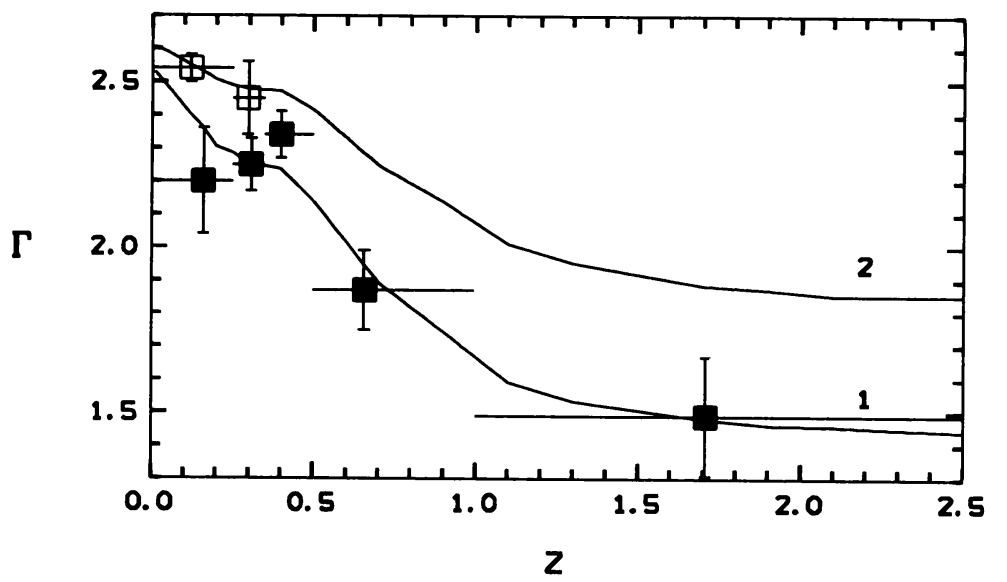

Fig. 1. Mean photon index in redshift bins plotted as a function of the redshift. Black and white symbols are for radio-loud and radio quiet-sources, respectively. The lines show the results of the simulations of the redshift effect on power law plus black body model spectra. The two models differ only in the photon index of the power law component.

373

T. J.-L. Courvoisier and A. Blecha: Multi-Wavelength Continuum Emission of AGN, 373.

(C) 1994 IAU. Printed in the Netherlands. 